\title{
Methicillin-Resistant Staphylococcus aureus and Candida albicans Secondary Bloodstream Co-Infection in a Patient with Tubular Oesophageal Duplication
}

\author{
Marta Carreira, Joana Pimenta \\ Internal Medicine Department, Centro Hospitalar Universitário de São João, Porto, Portugal
}

Received: 09/09/2020

Accepted: 07/10/2020

Published: $30 / 10 / 2020$

How to cite this article: Carreira M, Pimenta J. Methicillin-resistant Staphylococcus aureus and Candida albicans secondary bloodstream co-infection in a patient with tubular oeseophageal duplication. EJCRIM 2020;7: doi:10.12890/2020_001991.

Conflicts of Interests: The Authors declare that there are no competing interests.

This article is licensed under a Commons Attribution Non-Commercial 4.0 License

\section{ABSTRACT}

Gastrointestinal duplications are rare congenital anomalies usually found incidentally in asymptomatic adult patients. We report a case of methicillin-resistant Staphylococcus aureus and Candida albicans secondary bloodstream co-infection in a 57-year-old male patient with a communicating tubular oesophageal duplication. The patient completed 21 days of medical treatment with vancomycin and anidulafungin and remained well without any complications, over 2 years of follow-up.

\section{LEARNING POINTS}

- Tubular oesophageal duplications are rare and often asymptomatic.

- Candida spp. seem to facilitate Staphylococcus aureus concomitant infection.

- Bloodstream infection due to uncommon agents should prompt further and exhaustive investigation of the source of infection.

\section{KEYWORDS}

Oesophageal duplication, Staphylococcus aureus, Candida albicans

\section{INTRODUCTION}

Gastrointestinal duplications are uncommon congenital anomalies ${ }^{[1]}$. The estimated incidence of congenital oesophageal duplications (ODs) is $1: 8,200$, with male sex predominance ${ }^{[2]}$. Most ODs are found incidentally in asymptomatic patients; when symptomatic, the most common presentation is respiratory symptoms in early childhood ${ }^{[3]}$.

We report a rare case of methicillin-resistant Staphylococcus aureus (MRSA) and Candida albicans (C. albicans) bloodstream infection in a patient with a communicating tubular OD.

\section{CASE DESCRIPTION}

A 57-year-old male patient was admitted to our hospital emergency department because of fever. He had been recently hospitalized due to fever and small-volume pleural effusion, interpreted as community-acquired pneumonia. He was treated empirically with ceftriaxone and azithromycin, and $>40 \mathrm{mg}$ of prednisolone for 5 days. No blood cultures were drawn at the time. His past medical history was positive for hypertension, alcoholism (>130 g/day) and he had a 45-pack-year history of cigarette smoking. The patient had no dysphagia or chronic cough. 
On admission, his physical examination revealed absent lung sounds on the lower third of the right pulmonary field, tachycardia (120 bpm) and fever $\left(38.5^{\circ} \mathrm{C}\right)$. The results of arterial blood gas analysis were $\mathrm{pH} 7.515, \mathrm{pCO}_{2} 25.4 \mathrm{mmHg}, \mathrm{pO}_{2} 66.9 \mathrm{mmHg}$ and $\mathrm{HCO}^{3} 20.0 \mathrm{mmol} / \mathrm{l}$. His laboratory tests revealed: white blood cell count of $30,050 \times 10^{9} / \mathrm{l}(88.8 \%$ neutrophils), serum creatinine $1.41 \mathrm{mg} / \mathrm{dl}$, urea $61 \mathrm{mg} / \mathrm{dl}$ and C-reactive protein $177.6 \mathrm{~g} / \mathrm{dl}$.

The patient was then hospitalized for further investigation and treatment. Blood cultures were obtained and empiric antibiotherapy for hospital-acquired pneumonia was started with piperacillin/tazobactam. After MRSA and C. albicans were isolated, therapy was switched to vancomycin and anidulafungin. HIV testing was negative. Rheumatoid factor, antineutrophil cytoplasmic and antinuclear antibodies were normal. Serum levels of immunoglobulin G, A and M were normal. Infective endocarditis was ruled out by transthoracic echocardiography. Chest computed tomography (CT) showed a small-volume pleural effusion on the right pulmonary field, a thickening of the lower third of the oesophagus and an air-filled tract connecting the upper-medium oesophagus to its distal portion (Fig. 1). Pulmonary consolidations, interstitial infiltrates and cavitation were absent. An upper endoscopy (Fig. 2) showed 3 fistulous openings to a "second oesophagus" filled with mucopurulent material where MRSA and C. albicans were also isolated. Acid-fast bacilli were not detected on microscopic or cultural examination and polymerase chain reaction testing for Mycobacterium tuberculosis infection was negative. Gastric biopsy showed no signs of granulomatous disease and colonoscopy was normal.
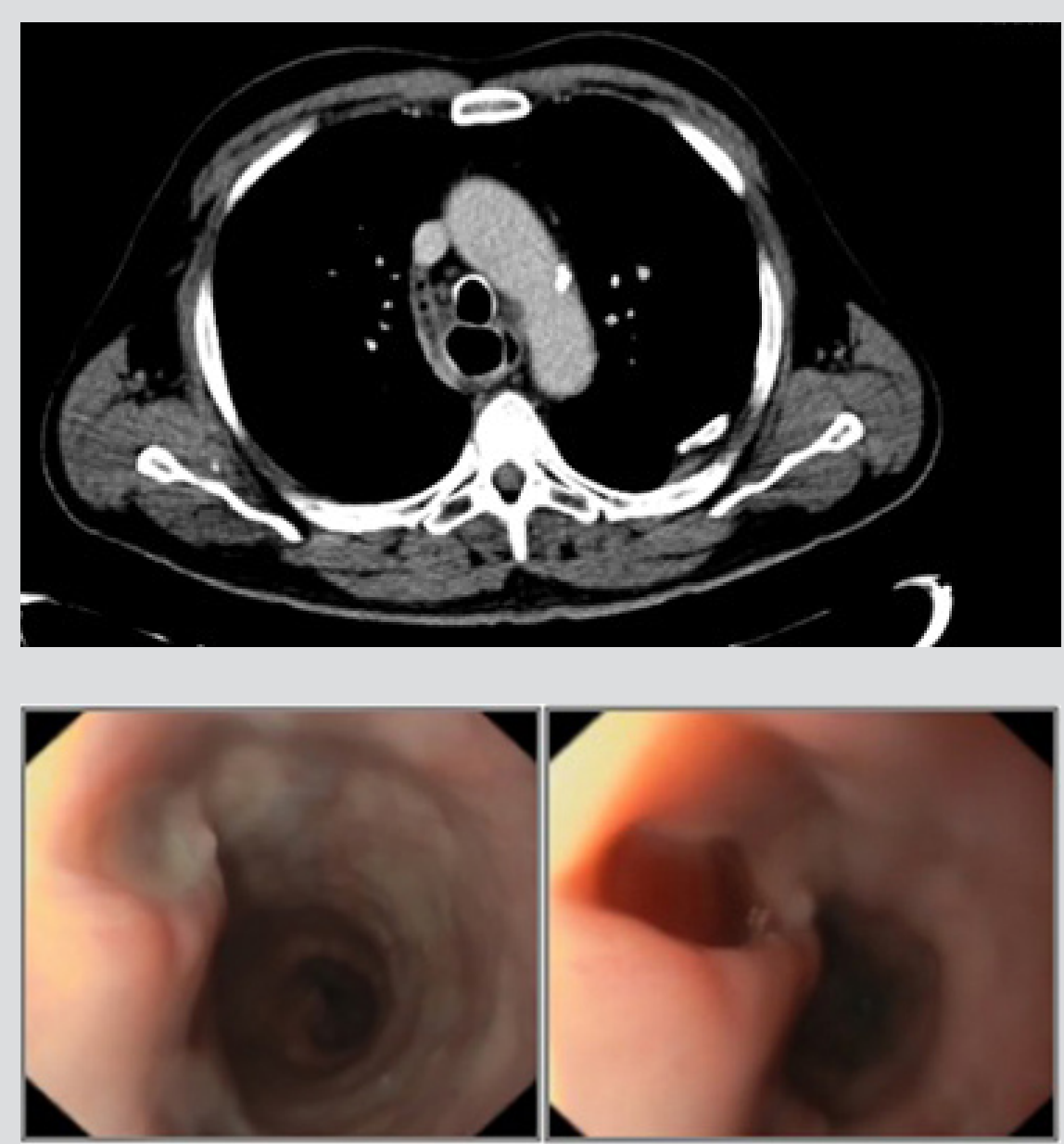

Figure 1. Chest CT showing an air-filled tract to the right of the "true" oesophagus

Figure 2. Upper endoscopy showing fistula openings to a "second oesophagus"

After discussion with our general surgery team, a conservative approach was preferred. The patient completed 21 days of medical treatment with vancomycin and anidulafungin. An upper endoscopy was repeated and showed no signs of infection. Videofluoroscopic modified barium swallow assessment revealed contrast leakage to the duplicate oesophagus confirming OD (Fig. 3). The patient was then discharged and remained well without any complications of OD, over 2 years of follow-up. 


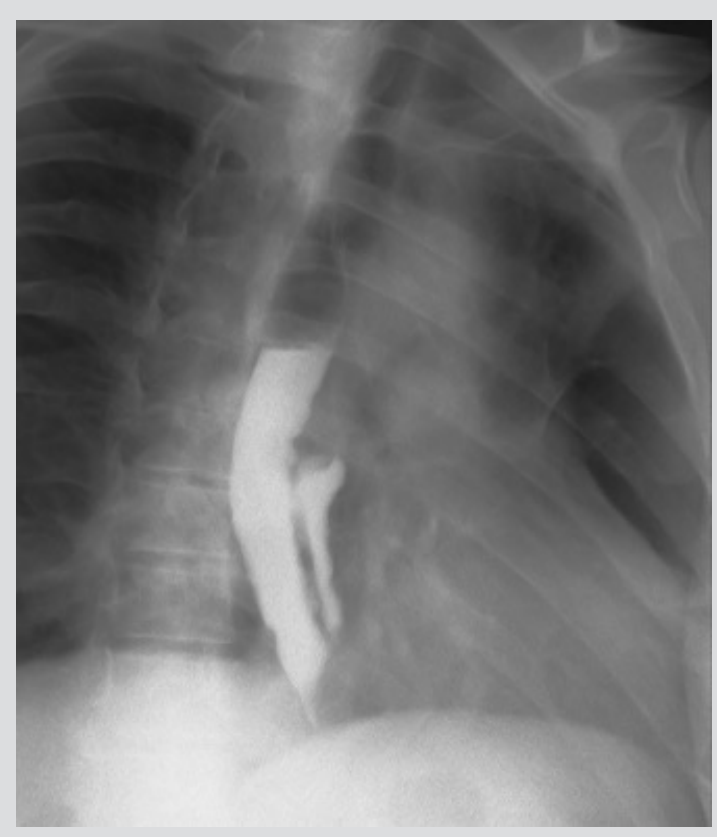

Figure 3. Videofluoroscopic modified barium swallow assessment revealed contrast leakage to the duplicate oesophagus

\section{DISCUSSION}

OD is divided into 2 types: cystic and tubular, although the cystic variant is much more common ${ }^{[2]}$. Duplications occur due to a defect in the tubulation of the oesophagus, in the sixth week of gestation; usually in the mid- and lower third of the oesophagus ${ }^{[4]}$.

Tubular duplications are typically reported in late childhood or adulthood, highlighting their asymptomatic nature ${ }^{[3]}$. To the best of our knowledge, an MRSA and C. albicans bloodstream infection secondary to infection of a duplicated oesophagus in an adult has never been previously described.

S. aureus is typically a non-invasive commensal of the oral cavity and nasopharynx ${ }^{[5]}$. Likewise, C. albicans frequently colonizes host mucosae $^{[6]}$. Co-isolation of S. aureus and C. albicans has been previously reported ${ }^{[7-9]}$.

In vitro studies show that $S$. aureus binds specifically to the hyphal form of $C$. albicans and not to the yeast form ${ }^{[10]}$. In the setting of immune dysfunction, C. albicans can transition from commensal to pathogen, switching from yeast to hyphal morphology with the ability to penetrate tissue, which is speculated to facilitate $S$. aureus concomitant infection ${ }^{[11]}$.

Risk factors for invasive C. albicans infection are well established ${ }^{[12]}$. Our patient had 2 predisposing factors that could facilitate $C$. albicans infection: an OD and treatment with high-dose glucocorticoids, which in turn could have promoted MRSA infection. The previous communityacquired pneumonia diagnosis could be questioned. The patient was initially admitted due to fever, elevated inflammatory markers and a small pleural effusion on x-ray. No blood cultures were drawn at the time. Later thoracic CT showed no pulmonary consolidations or interstitial infiltrates.

The management of OD is dependent on its type and size, and the severity of the symptoms. Complete resection is a well-documented treatment for $\mathrm{OD}^{[3]}$. In our case, due to the number and location of fistulae and the length of the duplication, a total oesophagectomy was indicated but it was deemed to be excessively mutilating.

OD is a rare diagnosis in adults for whom there is no standardized approach, although most cases described were surgically managed. To the best of our knowledge, this is the first case of bloodstream infection secondary to infection of the duplicated oesophagus. Bloodstream infection due to uncommon agents in immunocompetent adults should prompt further and exhaustive investigation of the source. 


\section{REFERENCES}

1. El-Gohary Y, Gittes GK, Tovar JA. Congenital anomalies of the esophagus. Semin Pediatr Surg 2010;19(3):186-193.

2. Achildi O, Grewal H. Congenital anomalies of the esophagus. Otolaryngol Clin North Am 2007;40(1):219-244, viii.

3. Trappey AF 3rd, Hirose S. Esophageal duplication and congenital esophageal stenosis. Semin Pediatr Surg 2017;26(2):78-86.

4. Patiño Mayer J, Bettolli M. Alimentary tract duplications in newborns and children: diagnostic aspects and the role of laparoscopic treatment. World J Gastroenterol 2014;20(39):14263-14271.

5. Krismer B, Weidenmaier C, Zipperer A, Peschel A. The commensal lifestyle of Staphylococcus aureus and its interactions with the nasal microbiota. Nat Rev Microbiol 2017;15(11):675-687

6. Arendrup MC. Epidemiology of invasive candidiasis. Curr Opin Crit Care 2010;16(5):445-452.

7. Baena-Monroy T, Moreno-Maldonado V, Franco-Martínez F, Aldape-Barrios B, Quindós G, Sánchez-Vargas LO. Candida albicans, Staphylococcus aureus and Streptococcus mutans colonization in patients wearing dental prosthesis. Med Oral Patol Oral Cir Bucal 2005;10 Suppl 1:E27-E39.

8. Cuesta Al, Jewtuchowicz V, Brusca MI, Nastri ML, Rosa AC. Prevalence of Staphylococcus spp and Candida spp in the oral cavity and periodontal pockets of periodontal disease patients. Acta Odontol Latinoam 2010;23(1):20-26.

9. Timsit JF, Cheval C, Gachot B, Bruneel F, Wolff M, Carlet J, et al. Usefulness of a strategy based on bronchoscopy with direct examination of bronchoalveolar lavage fluid in the initial antibiotic therapy of suspected ventilator-associated pneumonia. Intensive Care Med 2001;27(4):640-647.

10. Peters BM, Jabra-Rizk MA, Scheper MA, Leid JG, Costerton JW, Shirtliff ME. Microbial interactions and differential protein expression in Staphylococcus aureus-Candida albicans dual-species biofilms. FEMS Immunol Med Microbiol 2010;59(3):493-503.

11. Schlecht LM, Peters BM, Krom BP, Freiberg JA, Hänsch GM, Filler SG, et al. Systemic Staphylococcus aureus infection mediated by Candida albicans hyphal invasion of mucosal tissue. Microbiology (Reading) 2015;161(Pt 1):168-181.

12. Kullberg BJ, Arendrup MC. Invasive candidiasis. N Engl J Med 2015;373(15):1445-1456. 